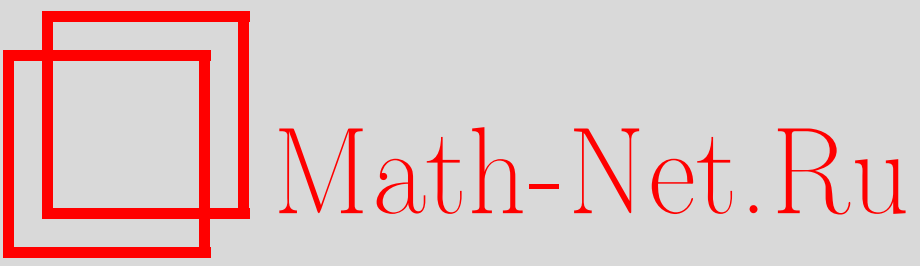

Я. А. Люлько, О распределении времени, проводимого марковской цепью на разных уровнях до момента достижения фиксированного состояния, Теория вероятн. и ее примен., 2011, том 56, выпуск 1, 167-176

DOI: https://doi.org/10.4213/tvp4333

Использование Общероссийского математического портала Math-Net.Ru подразумевает, что вы прочитали и согласны с пользовательским соглашением

http: //www. mathnet.ru/rus/agreement

Параметры загрузки :

IP: 54.84 .234 .179

26 апреля 2023 г., $15: 10: 42$ 
8. Ватанабэ C., Икэда Н. Стохастические дифференциальные уравнения и диффузионные процессы. М.: Наука, 1986, 448 с.

9. Dozzi M. Two-parameter stochastic processes. - Math. Res., 1991, v. 61, p. 17-43.

Поступила в редакцию 29.VII.2008

(c) 2011 г.

ЛЮЛЬКО Я.А.*

\title{
О РАСПРЕДЕЛЕНИИ ВРЕМЕНИ, ПРОВОДИМОГО МАРКОВСКОЙ ЦЕПЬЮ НА РАЗНЫХ УРОВНЯХ ДО МОМЕНТА ДОСТИЖЕНИЯ ФИКСИРОВАННОГО СОСТОЯНИЯ ${ }^{1)}$
}

\begin{abstract}
Данная работа посвящена исследованию вопроса о нахождении распределения времени пребывания однородной марковской цепи $Z=$ $\left(Z_{k}\right)_{k \geqslant 0}($ со счетным множеством состояний $E$ ) на разных уровнях фазового пространства до момента первого достижения фиксированного состояния $b \in E$.

Работа состоит из двух частей. В первой части с помощью строго марковского свойства показано, что в общем случае распределение времени пребывания будет геометрическим (с массой в нуле). В качестве примера рассмотрено скошенное случайное блуждание $S^{\alpha}=\left(S_{k}^{\alpha}\right)_{k \geqslant 0}$ с параметром $\alpha \in[0,1]$, для которого распределение времени пребывания найдено в явном виде.

Во второй части работы делается предельный переход от времени пребывания скошенного случайного блуждания к локальному времени скошенного броуновского движения $W^{\alpha}=\left(W_{t}^{\alpha}\right)_{t \geqslant 0}$. При этом основным инструментом для предельного перехода служит обобщенный принцип инвариантности Донскера-Прохорова.
\end{abstract}

Ключевые слова и фразы: марковская цепь, скошенное броуновское движение, локальное время, марковские моменты, принцип инвариантности Донскера-Прохорова.

\section{1. Введение.}

1.1. Пусть заданы вероятностное пространство $(\Omega, \mathscr{F}, \mathbf{P})$ и однородная марковская цепь $Z=\left(Z_{k}\right)_{k \geqslant 0}$ с множеством фазовых состояний $E=\left\{i_{0}, i_{1}, i_{2}, \ldots\right\}$, начальным распределением $p_{0}(i)=\mathbf{P}\left(Z_{0}=i\right)$ и переходными вероятностями $p_{i, j}=\mathbf{P}\left(Z_{n}=\right.$ $\left.j \mid Z_{n-1}=i\right), i, j \in E$. В качестве фильтрации рассмотрим естественную фильтрацию $\mathbb{F}=\left(\mathscr{F}_{n}\right)_{n \geqslant 0}, \mathscr{F}_{n}=\sigma\left(Z_{0}, \ldots, Z_{n}\right)$. Для каждого $x \in E$ определим меру $\mathbf{P}_{x}$ следующим образом: $\mathbf{P}_{x}(A)=\mathbf{P}\left(A \mid Z_{0}=x\right)$ для любого $A \in \mathscr{F}$. Положим

$$
N_{n}(a)=\sum_{k=0}^{n} \mathbb{I}\left(Z_{k}=a\right),
$$

где $a \in E$, и пусть $\tau_{b}=\inf \left\{k>0: Z_{k}=b\right\}$ для $b \in E$.

* Московский государственный университет им. М.В. Ломоносова, механикоматематический факультет, Ленинские горы, 119991 Москва, Россия; е-таil: yaroslav.lyulko@gmail.com

1) Работа выполнена при поддержке Фонда содействия отечественной науки. 


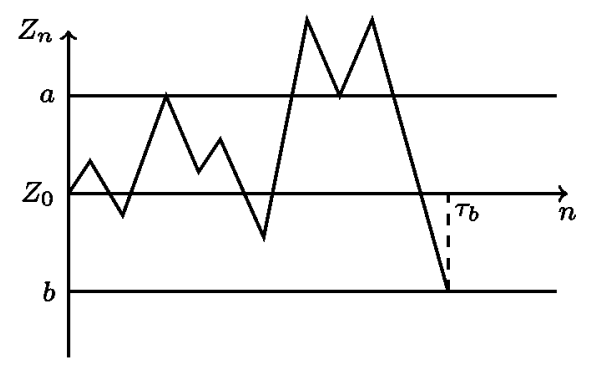

Рис. 1. Пересечение марковской цепью $\left(Z_{k}\right)_{k \geqslant 0}$ уровня $a$.

Мы будем интересоваться вопросом о распределении вероятностей случайной величины $N_{\tau_{b}}(a)$, которая есть число посещений состояния $a$ марковской цепью $Z$ до момента $\tau_{b}$ первого попадания цепи в состояние $b$. В теореме 1 п. 2.1 находится распределение $N_{\tau_{b}}(a)$ в общем виде. Фактически, эта теорема является следствием того, что отрезки марковской цепи между последовательными попаданиями в состояние $a \in E$ не зависят друг от друга.

В качестве примера применения теоремы 1 в п. 2.2 рассматривается скошенное случайное блуждание $S^{\alpha}=\left(S_{k}^{\alpha}\right)_{k \geqslant 0}, S_{0}^{\alpha}=0$. Для него находятся все параметры распределения в явном виде. В частности, получаются обобщения ранее известных результатов. Так, в случае симметричного бернуллиевского случайного блуждания известно (см., например, [7, гл. I, $\S 10$, задача 11]), что $\mathbf{E} N_{\tau_{0}}(a)=1$ и не зависит от уровня $a>0$. С помощью теоремы 1 и предложения 1 из п. 2.2 можно показать, что для скошенного случайного блуждания при любом $b<0$ математическое ожидание $\mathbf{E} N_{\tau_{b}}(a)$ равно $2 \alpha|b| /(1-\alpha)$, т.е. также не зависит от уровня $a>0$. При этом распределение времени $N_{\tau_{b}}(a)$ в этом случае все же зависит от $a$ :

$$
\begin{aligned}
\mathbf{P}\left(N_{\tau_{b}}(a)=0\right)= & \frac{(1-\alpha) a}{(1-\alpha) a+\alpha|b|}, \\
\mathbf{P}\left(N_{\tau_{b}}(a)=k\right)= & \frac{\alpha(1-\alpha)|b|}{2((1-\alpha) a+\alpha|b|)^{2}} \\
& \times\left(1-\frac{1-\alpha}{2((1-\alpha) a+\alpha|b|)}\right)^{k-1}, \quad k=1,2, \ldots .
\end{aligned}
$$

Скошенное случайное блуждание замечательно тем, что оно аппроксимирует скошенное броуновское движение. В разделе 3 с помощью результатов работ [8], [10] делается предельный переход и устанавливается закон распределения локального времени $L_{\tau_{b}}^{a}\left(W^{\alpha}\right)$ скошенного броуновского движения $\left(W_{t}^{\alpha}\right)_{t \geqslant 0}$ в точке $a$ до момента первого достижения уровня $b$. Оказывается, распределение $L_{\tau_{b}}^{a}\left(W^{\alpha}\right)$ будет показательным (с массой в нуле). В частности, при $b<0<a$ имеем

$$
\begin{aligned}
& \mathbf{P}\left(L_{\tau_{b}}^{a}\left(W^{\alpha}\right)=0\right)=\mu a, \\
& p(x)=\frac{d \mathbf{P}\left(L_{\tau_{b}}^{a}\left(W^{\alpha}\right) \leqslant x\right)}{d x}=\frac{\alpha|b| \mu^{2}}{2(1-\alpha)} e^{-\mu x / 2}, \quad x>0,
\end{aligned}
$$

где $\mu=\left(a+\alpha(1-\alpha)^{-1}|b|\right)^{-1}$. Заметим, что как и в дискретном случае, $\mathbf{E} L_{\tau_{b}}^{a}\left(W^{\alpha}\right)=$ $2 \alpha|b| /(1-\alpha)$.

1.2. Известная теорема Леви утверждает, что броуновское движение $B=\left(B_{t}\right)_{t \geqslant 0}$ обладает следующим свойством: $(\sup B-B, \sup B) \stackrel{\text { law }}{=}(|B|, L(B))$, где $L(B)=$ $\left(L_{t}(B)\right)_{t \geqslant 0}$ - локальное время в нуле. В работах [3] и [9] устанавливается дискретный аналог теоремы Леви для случайного блуждания $S=\left(S_{k}\right)_{k \geqslant 0}$ (в [9] основным инструментом доказательства является дискретная версия формулы Ито, а доказательство 
в работе [3] основывается на совпадении переходных функций фигурирующих в теореме марковских процессов). А именно, $(\max S-S, \max S) \stackrel{\text { law }}{=}(|S-1 / 2|-1 / 2, L(S))$, где $L_{n}(S)=\sum_{i=1}^{n} \mathbb{I}\left\{\left(S_{i-1}-1 / 2\right)\left(S_{i}-1 / 2\right)<0\right\}-$ количество пересечений случайным блужданием уровня $1 / 2$. Процесс $\left(L_{n}(S)\right)_{n \geqslant 0}$ играет для случайного блуждания ту же роль, что и локальное время в нуле для броуновского движения. Поэтому авторы работ [3], [9] называют процесс $L(S)$ аналогом локального времени в дискретном случае.

Однако локальное время $\left(L_{t}^{a}(X)\right)_{t \geqslant 0}$ непрерывного семимартингала $X=\left(X_{s}\right)_{s} \geqslant 0$ в точке $a \in \mathbf{R}$ определяется из формулы Танака:

$$
\left|X_{t}-a\right|=\left|X_{0}-a\right|+\int_{0}^{t} \operatorname{sign}\left(X_{s}-a\right) d X_{s}+L_{t}^{a}(X), \quad t \geqslant 0 .
$$

Если переписать (2) для симметричного случайного блуждания (см., например, $[7$, гл. I, $\S 9$, задача 3]), то получим

$$
\left|S_{k}-a\right|=\left|S_{0}-a\right|+\sum_{i=1}^{k} \operatorname{sign}\left(S_{i-1}-a\right)\left(S_{i}-S_{i-1}\right)+N_{k-1}(a) .
$$

Поэтому с этой точки зрения можно считать, что число пересечений $N_{k}(0)$ уровня 0 служит дискретным аналогом локального времени броуновского движения в нуле. Как будет показано в разделе 3 на примере скошенного броуновского движения, этот подход является более естественным при предельном переходе к локальному времени диффузионных процессов.

Стоит отметить, что существует общая теория локального времени диффузионных процессов со временем жизни $\zeta$, основанная на теоремах Рэя-Найта. С ее помощью, например, в $[2$, гл. V] найдено распределение локального времени стандартного броуновского движения с линейным сносом до момента первого достижения уровня $b$. В настоящей работе демонстрируется другой подход, основанный на аппроксимации диффузионных процессов марковскими цепями с дальнейшим предельным переходом, основанным на обобщенном принципе инвариантности.

2. Распределение времени пребывания $N_{\tau_{b}}(a)$.

2.1. Основная теорема. Распределение времени пребывания $N_{\tau_{b}}(a)$ произвольной марковской цепи $Z=\left(Z_{k}\right)_{k \geqslant 0}$ со счетным множеством состояний $E$ устанавливается в следующей теореме.

Теорема 1. Если $a \in E u b \in E(b \neq a)$ таковы, ито $\mathbf{P}_{a}\left(\tau_{a}<\tau_{b}\right)<1$, то распределение времени $N_{\tau_{b}}(a)$ относительно мерь $\mathbf{P}_{x}$ задается формулами

$$
\begin{aligned}
& \mathbf{P}_{x}\left(N_{\tau_{b}}(a)=0\right)=1-\beta_{x}, \\
& \mathbf{P}_{x}\left(N_{\tau_{b}}(a)=k\right)=\beta_{x}\left(1-\beta_{a}\right) \beta_{a}^{k-1}, \quad k=1,2, \ldots,
\end{aligned}
$$

где $\beta_{x}=\mathbf{P}_{x}\left(\tau_{a}<\tau_{b}\right)$ для $x \in E \backslash\{a\}$. В частности, $\mathbf{E}_{x} N_{\tau_{b}}(a)=\beta_{x} /\left(1-\beta_{a}\right)$.

Д о к а з а т е л ь с т в о. Заметим, что каждый из моментов $\tau_{b}$ будет марковским относительно естественной фильтрации $\left(\mathscr{F}_{n}\right)_{n \geqslant 0}$, но он может не быть конечным $\mathbf{P}_{x}$-п.н. Поэтому нужно определить $N_{\tau_{b}}(a)$ на множестве $\left\{\omega: \tau_{b}(\omega)=\infty\right\}$. Так как последовательность $\left\{N_{n}(a)\right\}_{n \geqslant 1}$ неубывающая, то положим $N_{\tau_{b}}(a)(\omega):=$ $\sum_{k=1}^{\infty} \mathbb{I}\left(Z_{k}(\omega)=a\right)$ на множестве $\left\{\omega: \tau_{b}(\omega)=\infty\right\}$. Однако в этом случае случайная величина $N_{\tau_{b}}(a)$ может принимать значение $+\infty$. Покажем, что при выполнении условия $\mathbf{P}_{a}\left(\tau_{a}<\tau_{b}\right)<1$ вероятность события $\left\{N_{\tau_{b}}(a)=+\infty\right\}$ равна нулю относительно меры $\mathbf{P}_{x}$ для каждого $x$.

Утверждение 1. 1) Если $\mathbf{P}_{a}\left(\tau_{a}<\tau_{b}\right)<1$, mо $\mathbf{P}_{x}\left(N_{\tau_{b}}(a)=+\infty\right)=0$ для любого $x \in E$.

2) Ecлu $\mathbf{P}_{a}\left(\tau_{a}<\tau_{b}\right)=1, \operatorname{mo} \mathbf{P}_{x}\left(N_{\tau_{b}}(a)=+\infty\right)=\mathbf{P}_{x}\left(\tau_{a}<\tau_{b}\right)$. 
Д о к а з а т е ль с т в о. Рассмотрим следующие моменты: $\tau_{a}^{1}=\tau_{a}, \tau_{a}^{k}=$ $\inf \left\{m>\tau_{a}^{k-1}: Z_{m}=a\right\}, k \geqslant 2$. Тогда $\mathbf{P}_{x}\left(N_{\tau_{b}}(a)=+\infty\right)=\mathbf{P}_{x}\left(\tau_{a}^{k}<\tau_{b}\right.$ для всех $k)=\lim _{k \rightarrow \infty} \mathbf{P}_{x}\left(\tau_{a}^{k}<\tau_{b}\right)$.

Обозначим $r_{k}(x)=\mathbf{P}_{x}\left(\tau_{a}^{k}<\tau_{b}\right)$. Тогда $r_{1}(x)=\mathbf{P}_{x}\left(\tau_{a}<\tau_{b}\right)=\beta_{x}$. Докажем по индукции, что

$$
r_{k}(x)=\mathbf{P}_{x}\left(\tau_{a}<\tau_{b}\right)\left[\mathbf{P}_{a}\left(\tau_{a}<\tau_{b}\right)\right]^{k-1}=\beta_{x} \beta_{a}^{k-1} .
$$

Пусть формула (5) верна для $r_{k-1}(x)$. По определению, $r_{k}(x)=\mathbf{P}_{x}\left(\tau_{a}^{k}<\right.$ $\left.\tau_{b}\right)=\mathbf{P}_{x}\left(\tau_{a}<\tau_{b}, \ldots, \tau_{a}^{k}<\tau_{b}\right)$. Если выполнено событие $\left\{\tau_{a}<\tau_{b}\right\}$, то выполнено и событие $\left\{\tau_{a}<\infty\right\}$. Значит, на множестве $\left\{\tau_{a}<\tau_{b}\right\}$ определены процесс $\widetilde{Z}_{n}=Z_{n+\tau_{a}}$ и моменты $\widetilde{\tau}_{b}=\tau_{b}(\widetilde{Z})=\inf \left\{k>0: \widetilde{Z}_{k}=b\right\}, \widetilde{\tau}_{b}^{k}=\tau_{b}^{k}(\widetilde{Z})$. Тогда $\mathbf{P}_{x}\left(\tau_{a}^{k}<\tau_{b}\right)=\mathbf{P}_{x}\left(\tau_{a}<\tau_{b}, \widetilde{\tau}_{a}<\widetilde{\tau}_{b}, \ldots, \widetilde{\tau}_{a}^{k-1}<\widetilde{\tau}_{b}\right)$. Пользуясь обобщенным строго марковским свойством (см., например, [6, гл. VIII, § 2, теорема 2], [7, с. 328, гл. VIII, $\S 2$, задача 13]), получим $r_{k}(x)=\mathbf{P}_{x}\left(\tau_{a}<\tau_{b}, \widetilde{\tau}_{a}<\widetilde{\tau}_{b}, \ldots, \widetilde{\tau}_{a}^{k-1}<\widetilde{\tau}_{b}\right)=\mathbf{P}_{x}\left(\tau_{a}<\right.$ $\left.\tau_{b}\right) \mathbf{P}_{a}\left(\tau_{a}<\tau_{b}, \ldots, \tau_{a}^{k-1}<\tau_{b}\right)=\beta_{x} r_{k-1}(a)=\beta_{x} \beta_{a}^{k-1}$.

Отсюда видно, что если $\beta_{a}<1$, то $\mathbf{P}_{x}\left(N_{\tau_{b}}(a)=+\infty\right)=\lim _{k \rightarrow \infty} r_{k}(x)=0$. Если же $\beta_{a}=1$, то $\mathbf{P}_{x}\left(N_{\tau_{b}}(a)=+\infty\right)=\mathbf{P}_{x}\left(\tau_{a}<\tau_{b}\right)=\beta_{x}$. Утверждение 1 доказано.

Далее будем считать, что $\mathbf{P}_{a}\left(\tau_{a}<\tau_{b}\right)<1$. Для завершения доказательства теоремы 1 достаточно заметить, что определенная в доказательстве утверждения 1 вероятность $r_{k}(x)$ равна $\beta_{x} \beta_{a}^{k-1}=\mathbf{P}_{x}\left(\tau_{a}^{k}<\tau_{b}\right)=\mathbf{P}_{x}\left(N_{\tau_{b}}(a) \geqslant k\right)$, откуда для $k \geqslant 1$ имеем $\mathbf{P}_{x}\left(N_{\tau_{b}}(a)=k\right)=\beta_{x} \beta_{a}^{k-1}-\beta_{x} \beta_{a}^{k}=\beta_{x} \beta_{a}^{k-1}\left(1-\beta_{a}\right)$. Теорема 1 доказана.

3 а м е ч а н и е 1 . Если $\mathbf{P}_{a}\left(\tau_{a}<\tau_{b}\right)=1$, то в силу утверждения 1 и того, что $\mathbf{P}_{x}\left(N_{\tau_{b}}(a)=0\right)=\mathbf{P}_{x}\left(\tau_{b} \leqslant \tau_{a}\right)=1-\beta_{x}$, распределение $N_{\tau_{b}}(a)$ для любого $x \neq a$ является двуточечным:

$$
\mathbf{P}_{x}\left(N_{\tau_{b}}(a)=0\right)=1-\beta_{x}, \quad \mathbf{P}_{x}\left(N_{\tau_{b}}(a)=+\infty\right)=\beta_{x} .
$$

3 а м е ч ан и е 2. Случай $x=a$ отличается от рассмотренных в теореме 1 случаев тем, что начальным значением $N_{\tau_{b}}(a)$ будет 1 , а не 0 . Вид распределения (4) не изменится.

2.2. Случай скошенного случайного блуждания. Скошенным случайным блужданием с параметром $\alpha \in[0,1]$ будем называть однородную марковскую цепь $S^{\alpha}=\left(S_{k}^{\alpha}\right)_{k \geqslant 0}, S_{0}^{\alpha}=0$, с множеством состояний $\mathbf{Z}$ и переходной функцией

$$
p_{i, j}=\mathbf{P}\left(S_{n}=j \mid S_{n-1}=i\right)= \begin{cases}\alpha, & \text { если } i=0, j=1, \\ 1-\alpha, & \text { если } i=0, j=-1, \\ 1 / 2, & \text { если } i \neq 0,|i-j|=1, \\ 0 & \text { иначе. }\end{cases}
$$

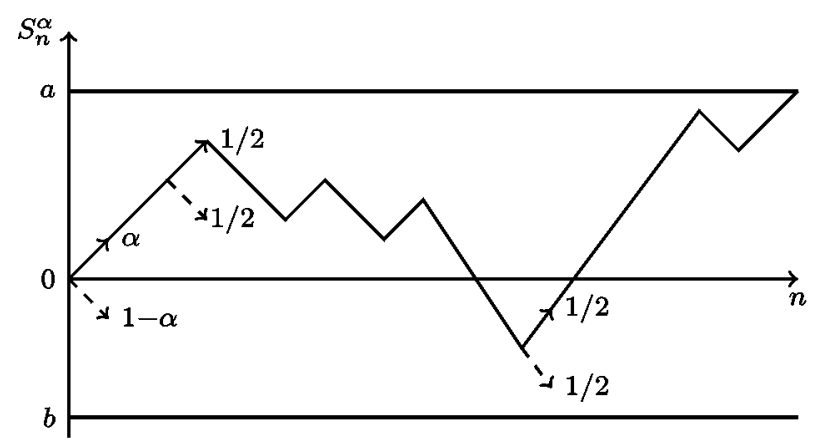

Рис. 2. Траектория скошенного случайного блуждания $\left(S_{k}^{\alpha}\right)_{k \geqslant 0}$.

Применив теорему 1 к данному процессу, получим распределение времени пребывания $N_{\tau_{b}}(a)$ на уровне $a$ вида $(4)$. Без ограничения общности будем считать, что 
$a \geqslant 0, b \in \mathbf{Z}, b \neq a$. Чтобы получить явный вид распределения, необходимо вычислить параметры $\beta_{x}$ и $\beta_{a}$. При этом нужно учесть, что в рассматриваемом случае $x=0$ и $\mathbf{P}_{0}=\mathbf{P}$.

Предложение 1. Для скошенного случайного блуждания $\left(S_{k}^{\alpha}\right)_{k \geqslant 0}$ параметры $\beta=\beta_{0}=\mathbf{P}\left(\tau_{a}<\tau_{b}\right)$ u $\beta_{a}=\mathbf{P}_{a}\left(\tau_{a}<\tau_{b}\right)$ при $a \geqslant 0, b \in \mathbf{Z} \backslash\{a\}$ определяются из mабл. 1 .

\section{Таблица 1}

\begin{tabular}{|c|c|c|c|c|c|}
\hline & $b<0<a$ & $b=0$ & $b>a>0$ & $a=0<b$ & $a=0>b$ \\
\hline$\beta$ & $\frac{\alpha|b|}{(1-\alpha) a+\alpha|b|}$ & $\frac{\alpha}{a}$ & 1 & $1-\frac{\alpha}{b}$ & $1-\frac{1-\alpha}{|b|}$ \\
\hline$\beta_{a}$ & $1-\frac{1-\beta}{2 a}$ & $1-\frac{1}{2 a}$ & $1-\frac{1}{2(b-a)}$ & $1-\frac{\alpha}{b}$ & $1-\frac{1-\alpha}{|b|}$ \\
\hline
\end{tabular}

Д о к а $з$ а т е л ь с т в о. 1) Рассмотрим сначала самый содержательный случай $b<0<a$. Предположим на время, что скошенное случайное блуждание может выходить из любой точки $S_{0}^{\alpha}=n$. Введем моменты $\gamma_{a}=\inf \left\{k \geqslant 0: S_{k}^{\alpha}=a\right\}$, $\gamma_{b}=\inf \left\{k \geqslant 0: S_{k}^{\alpha}=b\right\}$ и, пользуясь марковским свойством процесса $S^{\alpha}$, составим разностные уравнения для последовательности $f_{n}=\mathbf{P}_{n}\left(\gamma_{a}<\gamma_{b}\right), b \leqslant n \leqslant a$. При этом ясно, что полученные уравнения только в точке $n=0$ будут отличаться от соответствующих уравнений для симметричного случайного блуждания (которые известны, например, из $[5$, гл. $14, \S 2])$. Действительно, имеем

$$
\left\{\begin{array}{l}
f_{a}=1 \\
f_{n}=\frac{1}{2} f_{n-1}+\frac{1}{2} f_{n+1}, \quad b<n<a, \quad n \neq 0 \\
f_{0}=\alpha f_{1}+(1-\alpha) f_{-1}, \\
f_{b}=0 .
\end{array}\right.
$$

Решая полученную однородную систему, приходим к следующим уравнениям:

$$
\begin{cases}f_{n}=1+\left(1-f_{0}\right)(n-a) a^{-1}, & 0<n<a, \\ f_{n}=-f_{0}(n-b) b^{-1}, & b<n<0, \\ f_{0}=\alpha f_{1}+(1-\alpha) f_{-1}, & \end{cases}
$$

из которых находим $f_{0}=\mathbf{P}\left(\gamma_{a}<\gamma_{b}\right)=\mathbf{P}\left(\tau_{a}<\tau_{b}\right)$ и $\beta_{a}=1 / 2+f_{a-1} / 2$.

2) Остальные случаи либо доказываются аналогично предыдущему, либо следуют из уже известных (например, из [5]) результатов для симметричного случайного блуждания. Так, если $b=0$, то $\beta=\alpha \mathbf{P}_{1}\left(\tau_{a}<\tau_{0}\right)=\alpha / a$, так как вероятность $\mathbf{P}_{1}\left(\tau_{a}<\tau_{0}\right)$ одна и та же как для симметричного, так и для скошенного случайного блуждания. Из тех же соображений $\beta_{a}=1-1 /(2 a)$ при $b=0$ и $\beta_{a}=1-1 /(2 b-2 a)$ при $b>a>0$. Случай $a=0, b>0$ сводится к случаю $b=0$, а при $a=0, b<0$ имеем $\mathbf{P}\left(\tau_{0}<\tau_{b}\right)=\left.\mathbf{P}\left(\tau_{0}<\tau_{-b}\right)\right|_{\alpha \leftrightarrow(1-\alpha)}=1-(1-\alpha) /|b|$.

Предложение 1 доказано.

Подставляя значения параметров $\beta$ и $\beta_{a}$ в (4), найдем распределение времени посещения $N_{\tau_{b}}(a)$ в явном виде. В частности, при $b<0<a$ получим (1).

3. Предельный переход к локальному времени $L_{\tau_{b}}^{a}\left(W^{\alpha}\right)$ скошенного броуновского движения. Процесс скошенного броуновского движения был впервые введен в книге К. Ито и Г. Маккина [4], а затем подробнее изучен в работах [10] и [11]. Так, в работе [10] показано, что скошенное броуновское движение $W^{\alpha}=\left(W_{t}^{\alpha}\right)_{t \geqslant 0}$ с 
параметром $\alpha \in[0,1]$ является непрерывным семимартингалом, удовлетворяющим стохастическому уравнению

$$
X_{t}=X_{0}+B_{t}+(2 \alpha-1) L_{t}^{0}(X)
$$

где $B=\left(B_{t}\right)_{t \geqslant 0}-$ стандартное броуновское движение, $L_{t}^{0}(X)-$ локальное время в нуле процесса $X$. Скошенное броуновское движение с параметром $\alpha=1 / 2$ совпадает со стандартным броуновским движением $\left(B_{t}\right)_{t \geqslant 0}$, а с параметром $\alpha=1-$ с модулем броуновского движения $\left(\left|B_{t}\right|\right)_{t \geqslant 0}$.

Нас же будет интересовать приближение скошенного броуновского движения и его локального времени процессами с дискретным временем. Так, в работе [10] установлена сходимость по распределению скошенного случайного блуждания $S^{\alpha}=\left(S_{k}^{\alpha}\right)_{k \geqslant 0}$ к скошенному броуновскому движению $W^{\alpha}=\left(W_{t}^{\alpha}\right)_{t \geqslant 0}$, а именно,

$$
\left(n^{-1 / 2} S_{[n t]}^{\alpha}, t \geqslant 0\right) \stackrel{\text { Law }}{\longrightarrow}\left(W_{t}^{\alpha}, t \geqslant 0\right)
$$

в пространстве Скорохода $D[0, \infty)$. Однако для того, чтобы получить распределение локального времени $L_{\tau_{b}}(a)=L_{\tau_{b}}^{a}\left(W^{\alpha}\right)$ в точке $a$ до момента $\tau_{b}=\tau_{b}\left(W^{\alpha}\right)$, сходимости одномерных процессов нам недостаточно. Понадобится обобщение результата (6), полученное в работе [8].

На протяжении всего раздела договоримся использовать одно и то же обозначение $\tau_{b}$ для момента первого выхода на границу как для дискретных, так и для непрерывных процессов. Так, при рассмотрении $N_{\tau_{b}}(a)$ мы считаем, что $\tau_{b}=\tau_{b}\left(S^{\alpha}\right)=\inf \{k>$ 0: $\left.S_{k}^{\alpha}=b\right\}$, а в случае $L_{\tau_{b}}(a)$ считаем, что $\tau_{b}=\tau_{b}\left(W^{\alpha}\right)=\inf \left\{t \geqslant 0: W_{t}^{\alpha}=b\right\}$.

Основным результатом данного раздела является следующая теорема.

Теорема 2. Локальное время $L_{\tau_{b}}(a)=L_{\tau_{b}}^{a}\left(W^{\alpha}\right)$ скошенного броуновского движения $W^{\alpha}=\left(W_{t}^{\alpha}\right)_{t \geqslant 0}$ на уровне $a \geqslant 0$ до момента первого достижения состояния $b \neq$ а имеет показательное распределение ( $с$ массой в нуле), плотность $p(x)=d \mathbf{P}\left(L_{\tau_{b}}(a) \leqslant x\right) / d x$ которого определяется из табл. 2.

Таблица 2

\begin{tabular}{|c|c|c|c|c|}
\hline & $\mathbf{P}\left(L_{\tau_{b}}(a)=0\right)$ & $p(x), x>0$ & $\mathbf{E} L_{\tau_{b}}(a)$ & $\mu$ \\
\hline$a=0, b>0$ & 0 & $\mu e^{-\mu x}$ & $b \alpha^{-1}$ & $\alpha b^{-1}$ \\
\hline$a=0, b<0$ & 0 & $\mu e^{-\mu x}$ & $|b|(1-\alpha)^{-1}$ & $(1-\alpha)|b|^{-1}$ \\
\hline$b<0<a$ & $\mu a$ & $\frac{\alpha|b| \mu^{2}}{2(1-\alpha)} e^{-\mu x / 2}$ & $\frac{2 \alpha|b|}{1-\alpha}$ & $\frac{1}{a+\alpha(1-\alpha)^{-1}|b|}$ \\
\hline$b>a>0$ & 0 & $\mu e^{-\mu x}$ & $2(b-a)$ & $1 /(2 b-2 a)$ \\
\hline
\end{tabular}

Д о к а з а т е л ь с т в о. Рассмотрим процессы $X^{n}=\left(X_{t}^{n}\right)_{t \geqslant 0}$ и $Y^{n}=\left(Y_{t}^{n}\right)_{t \geqslant 0}$, определенные следующим образом: в рациональных точках $X_{k / n}^{n}=n^{-1 / 2} S_{k}^{\alpha}, Y_{k / n}^{n}=$ $n^{-1 / 2} \sum_{i=1}^{k} \operatorname{sign}\left(X_{(i-1) / n}^{n}\right)\left(S_{i}^{\alpha}-S_{i-1}^{\alpha}\right), k \in \mathbf{Z}_{+}$, а траектории $\left(X_{t}^{n}\right)_{t \geqslant 0}$ и $\left(Y_{t}^{n}\right)_{t \geqslant 0}$ являются ломаными с узлами $\left(k / n, X_{k / n}^{n}\right),\left(k / n, Y_{k / n}^{n}\right), k \geqslant 0$. В итоге для каждого $n \in \mathbf{N}$ имеем $X_{t}^{n}=n^{-1 / 2} S_{[n t]}+n^{-1 / 2}\left(S_{[n t]+1}^{\alpha}-S_{[n t]}^{\alpha}\right)$. Аналогичное выражение можно написать и для $Y_{t}^{n}$.

В работе [8] доказан обобщенный принцип инвариантности, согласно которому

$$
\left(X_{t}^{n}, Y_{t}^{n}, t \geqslant 0\right) \stackrel{\text { Law }}{\longrightarrow}\left(W_{t}^{\alpha}, \int_{0}^{t} \operatorname{sign}\left(W_{s}^{\alpha}\right) d W_{s}^{\alpha}, t \geqslant 0\right)
$$

при $n \rightarrow \infty$ в пространстве непрерывных функций $C[0, \infty)$. Используя этот результат, установим сходимость числа посещений состояния $a$ к локальному времени скошенного броуновского движения до момента $\tau_{b}$. Для этого применим дискретную версию 
формулы Танака (3) к скошенному случайному блужданию $S^{\alpha}=\left(S_{k}^{\alpha}\right)_{k \geqslant 0}$. Для каждого $a \in \mathbf{Z}_{+}$имеем

$$
\left|S_{k}^{\alpha}-a\right|=\left|S_{0}^{\alpha}-a\right|+\sum_{i=1}^{k} \operatorname{sign}\left(S_{i-1}^{\alpha}-a\right)\left(S_{i}^{\alpha}-S_{i-1}^{\alpha}\right)+\widetilde{N}_{k}(a)
$$

где $\widetilde{N}_{k}(a)=\sum_{i=0}^{k-1} \mathbb{I}\left(S_{i}^{\alpha}=a\right)=N_{k-1}(a)$. Пусть теперь $a \in \mathbf{R}_{+}$- любое неотрицательное число. Сделав замену $a \mapsto\lceil a \sqrt{n}\rceil$, разделив обе части (8) на $\sqrt{n}$ и обозначив $a_{n}=n^{-1 / 2}\lceil a \sqrt{n}\rceil$, получим:

$$
\begin{aligned}
& \left|n^{-1 / 2} S_{k}^{\alpha}-a_{n}\right|=\left|n^{-1 / 2} S_{0}^{\alpha}-a_{n}\right| \\
& \quad+n^{-1 / 2} \sum_{i=1}^{k} \operatorname{sign}\left(n^{-1 / 2} S_{i-1}^{\alpha}-a_{n}\right)\left(S_{i}^{\alpha}-S_{i-1}^{\alpha}\right)+n^{-1 / 2} \widetilde{N}_{k}(\lceil a \sqrt{n}\rceil) .
\end{aligned}
$$

Введем новый процесс $\left(\widetilde{N}_{t}^{n}\left(a_{n}\right)\right)_{t \geqslant 0}$ по аналогии с процессами $\left(X_{t}^{n}\right)_{t \geqslant 0}$ и $\left(Y_{t}^{n}\right)_{t \geqslant 0}$ : $\widetilde{N}_{k / n}^{n}\left(a_{n}\right)=n^{-1 / 2} \sum_{i=0}^{k-1} \mathbb{I}\left(X_{i / n}^{n}=a_{n}\right)=n^{-1 / 2} N_{k-1}(\lceil a \sqrt{n}\rceil)$, а $\tilde{N}_{t}^{n}\left(a_{n}\right)$ является ломаной с узлами $\left(k / n, \widetilde{N}_{k / n}^{n}\right)_{k \geqslant 0}$. Так как $X_{k / n}^{n}=n^{-1 / 2} S_{k}^{\alpha}$, то

$$
\left|X_{k / n}^{n}-a_{n}\right|=\left|X_{0}^{n}-a_{n}\right|+\frac{1}{\sqrt{n}} \sum_{i=1}^{k} \operatorname{sign}\left(X_{(i-1) / n}^{n}-a_{n}\right)\left(S_{i}^{\alpha}-S_{i-1}^{\alpha}\right)+\widetilde{N}_{k / n}^{n}\left(a_{n}\right) .
$$

Обозначим $\widehat{X}_{t}^{n}=X_{t}^{n}-a_{n}$ и введем кусочно-линейный процесс $\left(\widehat{Y}_{t}^{n}\right)_{t \geqslant 0}$, значения которого в рациональных точках равны $\widehat{Y}_{k / n}^{n}=n^{-1 / 2} \sum_{i=1}^{k} \operatorname{sign}\left(\widehat{X}_{(i-1) / n}\right)\left(S_{i}^{\alpha}-S_{i-1}^{\alpha}\right)$. Тогда (10) можно переписать в следующем виде:

$$
\left|\widehat{X}_{k / n}^{n}\right|=\left|\widehat{X}_{0}^{n}\right|+\widehat{Y}_{k / n}^{n}+\widetilde{N}_{k / n}^{n}\left(a_{n}\right) .
$$

Так как процессы $\left(\widehat{X}_{t}^{n}\right)_{t \geqslant 0},\left(\widehat{Y}_{t}^{n}\right)_{t \geqslant 0},\left(\widetilde{N}_{t}^{n}\left(a_{n}\right)\right)_{t \geqslant 0}$ определяются значениями в рациональных точках вида $(k / n)_{k \geqslant 0}$, то для всех $t \geqslant 0$ имеем

$$
\widetilde{N}_{t}^{n}=\left|\widehat{X}_{t}^{n}\right|-\left|\widehat{X}_{0}^{n}\right|-\widehat{Y}_{t}^{n}
$$

Из обобщенного принципа инвариантности (7) следует, что

$$
\left(\widehat{X}_{t}^{n}, \widehat{Y}_{t}^{n}, t \geqslant 0\right) \stackrel{\text { Law }}{\longrightarrow}\left(W_{t}^{\alpha}-a, \int_{0}^{t} \operatorname{sign}\left(W_{s}^{\alpha}-a\right) d W_{s}^{\alpha}, t \geqslant 0\right),
$$

а это вместе с (11) и формулой Танака (2) влечет слабую сходимость следующих двумерных процессов:

$$
\left(X_{t}^{n}, \widetilde{N}_{t}^{n}\left(a_{n}\right), t \geqslant 0\right) \stackrel{\text { Law }}{\longrightarrow}\left(W_{t}^{\alpha}, L_{t}^{a}\left(W^{\alpha}\right), t \geqslant 0\right)
$$

при $n \rightarrow \infty$ в пространстве $C[0, \infty)$. Оказывается, отсюда следует, что

$$
\tilde{N}_{\tau_{b}}^{n}\left(a_{n}\right) \stackrel{d}{\rightarrow} L_{\tau_{b}}^{a}\left(W^{\alpha}\right)
$$

для любого $b \in \mathbf{R}, b \neq a$.

Действительно, рассмотрим отображение $h: C[0, \infty) \times C[0, \infty) \rightarrow \mathbf{R}$, определенное следующим образом: $h(x, y)=y\left(\tau_{b}(x)\right)$, где $\tau_{b}(x)=\inf \{t \geqslant 0: x(t)=b\}$. Согласно [1, гл. I, §5, теорема 5.1], достаточно показать, что $\mathbf{P}\left\{\left(W^{\alpha}, L^{a}\left(W^{\alpha}\right)\right) \in D_{h}\right\}=0$, где $D_{h}$ - множество точек разрыва функции $h$. В нашем случае $D_{h}=\{(x, y) \in$ $C[0, \infty) \times C[0, \infty)$ : существует $\varepsilon>0$ такое, что $\left.\max _{0 \leqslant t \leqslant \tau_{b}+\varepsilon} W_{t}^{\alpha}=b\right\}$. Имеем

$$
\begin{aligned}
& \mathbf{P}\left\{\left(W^{\alpha}, L^{a}\left(W^{\alpha}\right)\right) \in D_{h}\right\}=\mathbf{P}\left(\exists \varepsilon>0: \max _{0 \leqslant s \leqslant \tau_{b}+\varepsilon} W_{s}^{\alpha}=b\right) \\
& \leqslant \mathbf{P}\left(\exists n: \max _{0 \leqslant s \leqslant \tau_{b}+1 / n} W_{s}^{\alpha}=b\right)
\end{aligned}
$$




$$
\begin{aligned}
& =\lim _{n \rightarrow \infty} \sum_{k=0}^{\infty} \mathbf{P}\left(\max _{0 \leqslant s \leqslant \tau_{b}+1 / n} W_{s}^{\alpha}=b, k<\tau_{b}+\frac{1}{n} \leqslant k+1\right) \\
& \leqslant \lim _{n \rightarrow \infty} \sum_{k=0}^{\infty} \mathbf{P}\left(\max _{0 \leqslant s \leqslant k} W_{s}^{\alpha}=b, k<\tau_{b}+\frac{1}{n} \leqslant k+1\right)=0,
\end{aligned}
$$

так как событие $\left\{\omega \in \Omega: \max _{0 \leqslant s \leqslant k} W_{s}^{\alpha}(\omega)=b\right\}$ имеет нулевую вероятность. Таким образом, условия теоремы для функции $h(x, y)$ выполнены, и мы получаем (13).

Выразим теперь левую часть (13) через время пребывания $N_{\tau_{\hat{b}}}(\widehat{a})$ скошенного случайного блуждания $S^{\alpha}$ на некотором уровне $\widehat{a}$ до момента первого достижения некоторого уровня $\widehat{b}$ (уровни $\widehat{a}$ и $\widehat{b}$ могут зависеть от $n$ ). А именно, покажем, что

$$
\frac{1}{\sqrt{n}} N_{\tau_{\lceil b \sqrt{n}\rceil}}(\lceil a \sqrt{n}\rceil) \stackrel{d}{\rightarrow} L_{\tau_{b}}^{a}\left(W^{\alpha}\right) .
$$

В самом деле, в силу того, что процесс $\widetilde{N}_{t}^{n}$ является кусочно-линейным с $\widetilde{N}_{k / n}^{n}=$ $n^{-1 / 2} N_{k-1}(\lceil a \sqrt{n}\rceil)$, для него справедливо представление $\widetilde{N}_{t}^{n}\left(a_{n}\right)=\widetilde{N}_{[n t] / n}^{n}\left(a_{n}\right)+$ $\left(\widetilde{N}_{([n t]+1) / n}^{n}\left(a_{n}\right)-\widetilde{N}_{[n t] / n}^{n}\left(a_{n}\right)\right)(n t-[n t])$. Отсюда следует, что

$$
\begin{aligned}
\widetilde{N}_{t}^{n}\left(a_{n}\right) & =\frac{1}{\sqrt{n}} \sum_{i=1}^{[n t]} \mathbb{I}\left(X_{(i-1) / n}^{n}=a_{n}\right)+\frac{n t-[n t]}{\sqrt{n}}\left(X_{[n t] / n}^{n}=a_{n}\right) \\
& =\frac{1}{\sqrt{n}} \sum_{i=1}^{[n(t+1 / n)]} \mathbb{I}\left(X_{(i-1) / n}^{n}=a_{n}\right)+\frac{n t-[n t]-1}{\sqrt{n}} \mathbb{I}\left(X_{[n t] / n}^{n}=a_{n}\right) \\
& =\frac{1}{\sqrt{n}} N_{\lceil n t\rceil}(\lceil a \sqrt{n}\rceil)+\gamma_{t}^{n} .
\end{aligned}
$$

Таким образом, $\tilde{N}_{t}^{n}\left(a_{n}\right)=n^{-1 / 2} N_{\lceil n t\rceil}(\lceil a \sqrt{n}\rceil)+\gamma_{t}^{n}$, где $\sup _{t \geqslant 0}\left|\gamma_{t}^{n}\right| \leqslant 3 / \sqrt{n}$. Тогда из теоремы Слуцкого и соотношения (13) получим

$$
\frac{1}{\sqrt{n}} N_{\left\lceil n \tau_{b}\right\rceil}(\lceil a \sqrt{n}\rceil) \stackrel{d}{\rightarrow} L_{\tau_{b}}^{a}\left(W^{\alpha}\right) .
$$

Из (12) следует, что момент $\tau_{b}$ есть момент первого выхода на уровень $b$ процесса $X^{n}$, т.е. $\tau_{b}=\tau_{b}\left(X^{n}\right)$. Тогда

$$
\left\lceil n \tau_{b}\left(X^{n}\right)\right\rceil=\inf \left\{k>0: X_{k / n}^{n} \geqslant b\right\}=\inf \left\{k>0: S_{k}^{\alpha}=\lceil b \sqrt{n}\rceil\right\}=\tau_{\lceil b \sqrt{n}\rceil}\left(S^{\alpha}\right),
$$

и поэтому (15) есть в точности (14). Тем самым искомая сходимость (14) времени пребывания $n^{-1 / 2} N_{\left\lceil n \tau_{b}\right\rceil}(\lceil a \sqrt{n}\rceil)$ скошенного случайного блуждания $\left(S_{k}^{\alpha}\right)_{k \geqslant 0}$ к локальному времени $L_{\tau_{b}}(a)=L_{\tau_{b}}^{a}\left(W^{\alpha}\right)$ скошенного броуновского движения $\left(W_{t}^{\alpha}\right)_{t \geqslant 0}$ установлена.

Перейдем теперь к конкретным вычислениям. Так как функция $f(x)=e^{-\lambda x}$ при каждом $\lambda \geqslant 0$ непрерывна и ограничена на $[0, \infty)$, то из слабой сходимости $(14)$ следует, что

$$
F_{n}(\lambda)=\mathbf{E} \exp \left\{-\frac{\lambda}{\sqrt{n}} N_{\tau_{\lceil b \sqrt{n}\rceil}}(\lceil a \sqrt{n}\rceil)\right\} \underset{n \rightarrow \infty}{\longrightarrow} \mathbf{E} e^{-\lambda L_{\tau_{b}}(a)}=F(\lambda)
$$

для каждого $a \geqslant 0, b \neq a$.

Из теоремы 1 мы можем найти производящую функцию времени пребывания $N_{\tau_{b}}(a)$. Она имеет следующий вид:

$$
\mathbf{E} s^{N_{\tau_{b}}(a)}=1-\frac{\beta_{0}(1-s)}{1-s \beta_{a}}, \quad s \in(0,1) .
$$

Делая в (17) замену $s \mapsto \exp \{-\lambda / \sqrt{n}\}, a \mapsto\lceil a \sqrt{n}\rceil, b \mapsto\lceil b \sqrt{n}\rceil$ и подставляя в производящую функцию найденные в п. 2.2 параметры $\beta_{0}$ и $\beta_{a}$, получим левую 
часть (16), а из нее предельным переходом преобразование Лапласа для $L_{\tau_{b}}(a)$. Результаты, получающиеся при различных значениях параметров $a$ и $b$, представлены в табл. 3.

Таблица 3

\begin{tabular}{|c|c|}
\hline & $F(\lambda)$ \\
\hline$a=0, b>0$ & $(1+\lambda b / \alpha)^{-1}$ \\
\hline$a=0, b<0$ & $(1+\lambda|b| /(1-\alpha))^{-1}$ \\
\hline$b<0<a$ & $\frac{(1-\alpha) a}{(1-\alpha) a+\alpha|b|}+\frac{\alpha|b|\left(1+2\left(a+\alpha(1-\alpha)^{-1}|b|\right) \lambda\right)^{-1}}{(1-\alpha) a+\alpha|b|}$ \\
\hline$b>a>0$ & $(1+2 \lambda(b-a))^{-1}$ \\
\hline
\end{tabular}

Отсюда следует, что распределение $L_{\tau_{b}}(a)$ во всех случах будет показательным (с массой в нуле), плотность которого задается табл. 2.

Проведем подробные вычисления в случае $b<0<a$. При других соотношениях на $a$ и $b$ преобразование Лапласа $F(\lambda)$ находится аналогично. Из табл. 1 параметры $\beta_{0}, \beta_{a \sqrt{n}}$ определяются следующим образом:

$$
\beta_{0}=\frac{\alpha|b|}{(1-\alpha) a+\alpha|b|}+o(1), \quad \beta_{a \sqrt{n}}=1-\frac{1-\beta_{0}}{2 a \sqrt{n}}+o(1) .
$$

Имеем:

$$
\begin{aligned}
F_{n}(\lambda)= & 1-\left(\frac{\alpha|b|}{(1-\alpha) a+\alpha|b|}+o(1)\right)\left(\frac{\lambda}{\sqrt{n}}+o\left(\frac{1}{\sqrt{n}}\right)\right) \\
& \times\left\{1-\left(1-\frac{\lambda}{\sqrt{n}}+o\left(\frac{1}{\sqrt{n}}\right)\right)\left(1-\frac{1-\beta_{0}}{2 a \sqrt{n}}+o(1)\right)\right\}^{-1} \\
= & 1-\left(\frac{\lambda}{\sqrt{n}} \frac{\alpha|b|}{(1-\alpha) a+\alpha|b|}+o\left(\frac{1}{\sqrt{n}}\right)\right)\left\{\frac{1}{\sqrt{n}}\left(\lambda+\frac{1-\beta_{0}}{2 a}+o(1)\right)\right\}^{-1} \\
\underset{n \rightarrow \infty}{\longrightarrow} & 1-\frac{\alpha|b|}{(1-\alpha) a+\alpha|b|} \lambda\left\{\lambda+\frac{1}{2\left(a+\alpha(1-\alpha)^{-1}|b|\right)}\right\}^{-1} \\
= & \frac{(1-\alpha) a}{(1-\alpha) a+\alpha|b|}+\frac{\alpha|b|}{(1-\alpha) a+\alpha|b|} \frac{1}{1+2\left(a+\alpha(1-\alpha)^{-1}|b|\right) \lambda} \\
= & \mathbf{E} e^{-\lambda L_{\tau_{b}}(a)}=F(\lambda) .
\end{aligned}
$$

Теорема 2 доказана.

Заметим, что при $\alpha=1 / 2$ мы получаем локальное время стандартного броуновского движения $W^{1 / 2}=B$, для которого распределение $L_{\tau_{b}}(a)$ известно (см., например, [2]).

Автор выражает признательность своему научному руководителю А. Н. Ширяеву за постановку задачи и внимание к работе.

\section{СПИСОК ЛИТЕРАТУРЫ}

1. Биллингсли П. Сходимость вероятностных мер. М.: Наука, 1977, 352 с.

2. Бородин А.Н., Салминен П. Справочник по броуновскому движению. СПб.: Лань, 2000, 639 c. 
3. Мищенко A. C. Дискретный процесс Бесселя и его свойства. - Теория вероятн. и ее примен., 2005, т. 50 , в. 4, с. 797-806.

4. Ито К., Маккин Г. Диффузионные процессы и их траектории. М.: Мир, 1968, $394 \mathrm{c}$.

5. Феллер В. Введение в теорию вероятностей и ее приложения, т. 1. М.: Книжный дом «Либроком», 2010, 528 с.

6. Ширяев А.Н. Вероятность-2. М.: МЦНМО, 2007, 416 с.

7. Ширяев А. Н. Задачи по теории вероятностей. М.: МЦНМО, 2006, 416 с.

8. Cherny A., Shiryaev A., Yor M. Limit behaviour of the «horizontal-vertical» random walk and some extensions of the Donsker-Prokhorov invariance principle. - Теория вероятн. и ее примен., 2002, т. 47, в. 3, с. 498-517.

9. Fujita T. A random walk analogue of Levy's theorem. - Studia Sci. Math. Hungar., 2008 , v. 45, № 2 , p. 223-233.

10. Harrison J. M., Shepp L. A. On skew Brownian motion. - Ann. Probab., 1981, v. 9, № 2 , p. 309-313.

11. Walsh J. B. A diffusion with a discontinuous local time. - Astérisque, 1978, v. 52-53, p. $37-45$.

Поступила в редакцию

26.XI.2009

Исправленный вариант

24.XII. 2010

(C) $2011 \Gamma$.

МУМИНОВ М. С.

\section{О ПРЕДЕЛЬНОМ РАСПРЕДЕЛЕНИИ \\ МАКСИМАЛЬНОГО УКЛОНЕНИЯ \\ ЭМПИРИЧЕСКОЙ ПЛОТНОСТИ РАСПРЕДЕЛЕНИЯ И ФУНКЦИИ РЕГРЕССИИ II.}

В работе для неизвестной плотности распределения $f(t), t \in \mathbf{R}^{\nu}$, случайного вектора $X \in \mathbf{R}^{\nu}$ и функции регрессии $r(t)=\mathbf{E}(Y \mid X=t)$ случайного вектора $(X, Y), X \in \mathbf{R}^{\nu}, Y \in \mathbf{R}^{1}$, построены непараметрические ядерные оценки $f_{n}(t)$ и $r_{n}(t)$ соответственно. Доказано, что распределение максимального уклонения этих оценок от истинных плотности распределения $f(t)$ и функции регрессии $r(t)$ стремится к двойному экспоненциальному закону при $n \rightarrow \infty$. С помощью построенных оценок найдена доверительная область для $f(t)$ и $r(t)$, отвечающая заданному коэффициенту доверия $\alpha(0<\alpha<1)$, и построен критерий для проверки гипотезы $H_{0}: f(t)=f_{0}(t)$ (соответственно $H_{0}^{\prime}: r(t)=r_{0}(t)$ ), где $f_{0}(t)-$ заранее заданная плотность распределения вероятностей, $r_{0}(t)$ - некоторая заданная функция.

Ключевые слова и фразы: последовательность случайных векторов, плотность распределения вероятностей, функция регрессии, непараметрические ядерные оценки, винеровский процесс с многомерным параметром, локальная стационарность случайных полей, доверительная область, проверка статистических гипотез.

* Институт математики и информационных технологий АН РУз, отдел теории вероятностей и математической статистики, ул. Ф. Ходжаева, 29, 100125, Ташкент, Узбекистан; e-mail: m.muhammad@rambler.ru 